\title{
Recommendation of Fashion Coordinates Considering TPO and Impression
}

\author{
Keisuke Kastura $_{1}$, Momoko Kato ${ }^{2}$ and Hiromitsu Simakawa ${ }^{1}$
}

\begin{abstract}
This paper proposes a method to recommend combination of clothes that give others the appropriate impression users want to give according to the TPO(Time, Place, and Occasion). This method calculates the impression of users' clothes based on ones in the websites. This method extracts clothes matching the impression websites consider suitable for the TPO. When users specify the TPO, this method recommends combination of the users' clothes which gives impression similar to impression of the clothes in the websites for the TPO. In the experiment, this method can recommend clothes with impression users want to give with $63 \%$ in the precision. With the method, users can go out with confidence, wearing clothes appropriate to target TPOs.
\end{abstract}

Keywords-Impression Regression Model, Impression Coordinates Space, Recommendation, TPO

\section{INTRODUCTION}

Many people preconceive an impression to predict the personality of others based on the combination of clothes[1][2]. Therefore, many people think they should give a good impression, wearing appropriate clothes in a particular situation[9]. When a person selects clothes, the person considers the impression from others. The impression the users want to give others varies with TPO(Time, Place, and Occasion). However, when users have poor knowledge of the situation, it is difficult to choose the clothes matching the TPO from a large variety of clothes. The users cannot objectively assess the strength of impression they want to give even though they have knowledge of the situation. Therefore, it is necessary to recommend clothes to users that give others the appropriate impression they want to give according to the TPO.

This method calculates the impression of the users' clothes based on the clothes in websites. This method extracts the clothes which gives impression the websites recommends for each TPO. For the TPO the user specifies, this method recommends combination of the users' clothes which give the impression similar to that of clothes on the websites. The paper shows an experimental result to prove the method recommends combination of clothes that give others the appropriate impression according to the TPO.

\section{RELATED STUDIES}

As previous studies of clothes recommendation, there is literature [5-8],[10]. The work in [8] proposed a system to recommend the clothes of impression that suit a particular

\footnotetext{
${ }^{1}$ Master Student, Ritsumeikan Unversity, Japan

${ }^{2}$ Master Student, Ritsumeikan Unversity, Japan
}

scene, using the colts in the websites and text data associated with those images. This work constructs combination of the clothes based on impression of one garment. However, it is not necessarily equivalent to impression of the garment and impression of coordination using the garment. For example, let us take the case of a coordinate of a white blouse of elegant impression and a denim pants. In this case, there is a possibility the elegance the white blouse gives is impaired by casual denim pants. Though, work in [8] has identified the impression of a garment, it does not take it into account until the strength of the clothes of the impression. Taking into account the impression, users can choose more accurately a coordinate having the impression they want to give. The work [5-7],[10] recommend clothes for a particular situation from the history of the past of clothes. They recommend clothes considering matching the TPO. However, they do not consider the impression of clothes. From the above discussion, the existing works include the following problems.

1. They do not take into account the impression of coordination.

2. Impression of a garment is not necessarily equivalent to that of a coordination using the garment.

They cannot recommend the coordination which gives impression suitable for a particular TPO.

\section{ReCOMMENDATION TECHNIQUE}

\section{A. Definition of the Impression and the TPO}

This paper proposes a method to recommend combination of clothes which give others the appropriate impression according to the TPO.

It is said that the impressions are classified in eight categories in fashion business[3]. Table1 shows the eight kinds of impression. The eight kinds of impression is composed of four pairs, each of which consists of two kinds of symmetric impression: ethnic and modern, romantic and mannish, elegance and active, sophisticate and country. We can consider TABLE I consists of 4 axes, each of which has the symmetric values. This work employs the eight kinds of impression in order to quantify impression of the garments.

The proposed method creates a coordinate space using four axes. This study refers to the four-dimensional coordinate space using the four axes as the impression space. This method regards romantic, elegance, sophisticate and modern as the positive direction in the impression space, while it regards mannish, active, country and ethnic as the negative.

This method regards seasons as the time elements of TPO. It takes the luxury of places to visit as the place element. It 
regards people the user meets in visited places as the occasion elements. It categorizes the season as two types; one consists of spring and summer, while the other autumn and winter. The luxury of the place is represented with three levels: higher, intermediate and lower. The people the user meets in visited places are modelled with the boyfriend, friends, and coworkers.

TABLE I: EIGHT IMPRESSIONS

\begin{tabular}{c|c}
\hline \hline Ethnic & Simple-Heatred Impression \\
\hline Romantic & Cute Impression \\
\hline Elegance & Elegant Impression \\
\hline $\begin{array}{c}\text { Sophisticat } \\
\mathrm{e}\end{array}$ & Urban or Adult-like Impression \\
\hline Modern & Intellectual Impression \\
\hline Mannish & Manly or Boyish Impression \\
\hline Active & Cheerful Impression \\
\hline Country & Rustic or Country-Style Impression \\
\hline
\end{tabular}

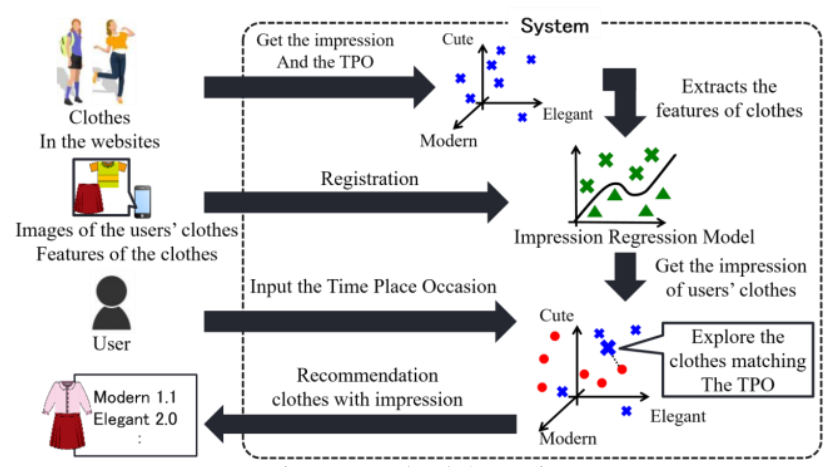

Fig. 1: Method Overview

\section{B. Recommendation Technique of Clothes}

The method calculates the impression of clothes from features of the users' clothes. An overview of the proposed method is shown in Fig.1.

First, the method calculates the impression of the garments of users, using images of the garments in the websites [4] as external standards. The matching TPO of garments on websites is obtained with a questionnaire given to the public. Based on the matching TPO of the garments on websites, the method associates the impression with the TPO. Based on the garments, the method constructs a model that predicts the impression of the garments from their features by the regression. This paper refers the constructed model as the Impression Regression Model. This model quantitatively calculates the impression the user intends to give by his or her clothes. Next, the user register features of his or her garments own to the system to get the quantitative impression.

Users specify the TPO when they want the recommendation. The method extracts all of the website's clothes matching the specified TPO. The method recommends user garments similar to the impression to the extracted clothes. The user chooses the clothes having the nearest impression to what he or she wants to give to others from the recommended clothes. Accordingly, this method recommends clothes to the users which give a good impression matching the specified TPO.

\section{Recommendation Technique of Clothes}

The method constructs the regression model to predict the impression of users' garments. How to construct regression model is shown in Fig. 2. The impression of coordination is formed by the combination of the various features of the garment. When we construct the regression model, garments in the website are used as training data. The method handles either pairs of tops and bottoms, or one piece. A pairs of a top and a bottom is one coordination, while a one-piece shall be a coordination with one garment.

Explanatory variables are features of tops and bottoms captured from garments in the websites. Features of garments mean their shape, colour, and length. Some of the indicators of one piece is shown in Table II. We choose the most applicable features for each item for the clothes using garments in the website. The combination of fitting feature quantity for each item is the explanatory variable of one of garments.

The objective variables are the impression obtained from the questionnaire results. The method uses neural networks to determine the impression of clothes.

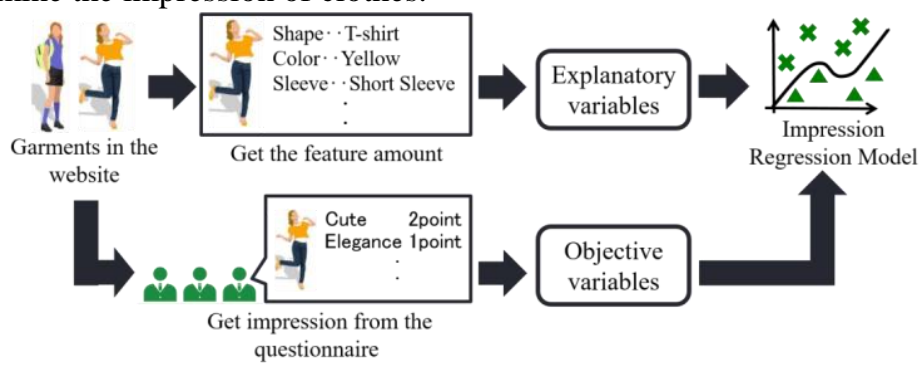

Fig. 2: Impression Regression Model

TABLE II: SOME Of THE INDICATORS OF THE ONE-PIECE

\begin{tabular}{c|cc}
\hline \hline $\begin{array}{l}\text { Kind of one } \\
\text { piece }\end{array}$ & One piece Length & Sleeve Length \\
\hline $\begin{array}{c}\text { One piece } \\
\text { Shirt one piece }\end{array}$ & $\begin{array}{c}\text { Short } \cdot \text { Mini } \\
\text { Tunic }\end{array}$ & Short Sleeve \\
Dress & Long & Half Sleeve \\
Long Sleeve \\
No sleeve
\end{tabular}

\section{Evaluation}

\section{A. Experimental outline}

We have experimented on two kinds of applications to verify the usefulness of this method. The purpose of our two experiments is summarized as follows. One is to verify whether it is possible for the method to recommend garments giving the impression users want to give based on a specified TPO. The other is to verify whether the method can recommend garments which actually give others the appropriate impression users want according to the TPO. We experiment with users who wear garments as examinees in the experiment of the first purpose, while the public people who see the users wearing garments as examinees of the second.

\section{B. Questionnaire Overview}

We have carried out experiments to verify the first experimental purposes, implementing an actual system. We performed two surveys described in Section 3.2 to build the 
system. In the first questionnaire, we investigate the impression of garments in the website. Subjects are 30 Japanese male and female students between 19 and 23 years old. For every garment in the website, subjects evaluated by 0 3 of 4 stages for eight impressions which ware described in Section 3.1. From the questionnaire results, we determined the value of the four impression in order to plot the garments in the website in the impression space. There are pairs of impression which constitute the symmetry like ethnic and modern. We calculate the difference between the absolute value of those two kinds of impression. As a result, we have four values of the impression. For example, among the eight obtained impressions, suppose the average value of the "Elegance" is 2.6, and the average value of the "Active" is 0.4. "Elegance" and "Active" is the impression which is symmetry on the same axis. Therefore, we regard the negative direction of the average value of the "active" as -0.4 . We calculate the value of the "Elegant - Active" axis as 2.2 from the sum of the two averages. We determined the value of the impression in the same way for the other three axes.

Next, we carried out questionnaires to get what garments in the website matches which TPO. Subjects are 25 people from 19 to 23 year old Japanese male and female students. The questionnaire was carried out using the TPO of each combination to one of garments. The questionnaire content is "Is this coordination appropriate coordination in the presented TPO?" Subjects answered "yes" and "no" to the questionnaire content. For example, in case of "Time: spring and summer, Place: luxury, Occasion: lover", subjects assessed garments whether is appropriate. Subjects evaluated a combination of all of the TPO for the same coordination. From the evaluation results, we calculated garments matching to each TPO. If the percentage of subjects answering "yes" is more than $80 \%$, the garment is defined as the garment matching the TPO.

\section{Regression Model Created using the Questionnaire Results}

We have created the impression regression model from the feature of coordination described in Section 3.2 and the impression of garments obtained from the questionnaire. We used four neural networks for each axis of the impression to create impression regression model. The four neural networks are shown in TABLE III. Description variables in the input layer represent the feature of clothing used for coordinates. On the basis of the category table of clothes in Section 3.3, they are 1 if the garment satisfies the condition, otherwise 0 . The intermediate layer of each axis are adopted the smallest number of error of the output results and correct data of the training data. Objective variable of the output layer is the value of the impression of garments acquired from the questionnaire. 80 pieces all of garments in the website were used as training data.

TABLE III: NEURAL NETWORK OF EACH AXIS

\begin{tabular}{l|c|c|c|c}
\hline \hline Impression Axis & $\begin{array}{l}\text { Layers } \\
\text { number }\end{array}$ & $\begin{array}{l}\text { Input } \\
\text { layer }\end{array}$ & $\begin{array}{l}\text { Middl } \\
\text { e layer }\end{array}$ & $\begin{array}{c}\text { Outpu } \\
\text { t layer }\end{array}$ \\
\hline \hline Ethnic - Modern & 3 & 112 & 5 & 1 \\
\hline Romantic - Mannish & 3 & 112 & 3 & 1 \\
\hline Elegance - Active & 3 & 112 & 3 & 1 \\
\hline
\end{tabular}

\begin{tabular}{l|l|l|l|l}
\hline $\begin{array}{l}\text { Sophisticate - } \\
\text { Country }\end{array}$ & 3 & 112 & 5 & 1 \\
\hline \hline
\end{tabular}

\section{First Experiment}

The firs experiment examines the first purpose. Users register the image of garments and the features of garments to the system. Users specify the TPO in recommendation. The system recommends the garments of users matching the TPO. Users answer a questionnaire for the recommended garments. The questionnaire content is "Do you think the impression of the recommended clothes is what you want to give to others in the specified TPO?"

\section{E. Second Experiment}

We verified the judgment of the public people on the impression of clothes recommended to users in the previous experiment. Subjects answered a questionnaire on the recommended garments. The questionnaire contents are "Do you think that the recommended garment match the specified TPO?" and "Do you think the impression of the recommended garment is appropriate?"

\section{F. Results}

Experiment results are shown in TABLEIV and TABLEV. In questionnaire content of the first experiment, this method could recommend clothes giving the impression that users want to give others with a $63.3 \%$ in the precision. Therefore, this method could recommend garment that give the impression the user wants to give others. In the former questionnaire of the second experiment, this method could recommend clothes matching the specified TPO with $40 \%$ in the precision. In the latter questionnaire, this method could recommend clothes giving the appropriate impression with $50 \%$ in the precision. If we take the logical product of the two questionnaires, the method could recommend clothes that give an impression appropriate to the TPO with $30 \%$ in the precision. Through the all of experiments, this method could recommend garments with which users can give others the appropriate impression they intend with $20 \%$ in the precision.

TABLE IV: EVALUATION OF THE EXPERIMENTAL 1

\begin{tabular}{|c|c|c|c|c|c|c|c|c|c|c|c|}
\hline Subject & A & B & $\mathrm{C}$ & $\mathrm{D}$ & $\mathrm{E}$ & $\mathrm{F}$ & $\mathrm{G}$ & $\mathrm{H}$ & I & $\mathbf{J}$ & sum \\
\hline $\begin{array}{l}\text { Evaluation } \\
\text { results }\end{array}$ & 1 & 3 & 3 & 1 & 2 & 3 & 1 & 2 & 1 & 2 & 19 \\
\hline
\end{tabular}

TABLE V: EVALUATION OF THE EXPERIMENTAL 2

\begin{tabular}{c|c|c|c}
\hline \hline Content & Content(1) & Content(2) & Content(1)(2) \\
\hline $\begin{array}{c}\text { The number of clothes } \\
\text { that meet the content }\end{array}$ & 12 & 15 & 9 \\
\hline \hline
\end{tabular}

\section{DISCUSSION}

\section{A. Recognition of the User in the TPO and Impressive}

This method could recommend garments which give others the appropriate impression users want to give according to the TPO with $20 \%$ in the precision in the experimental results. This means many of recommendation clothes could not give 
others the appropriate impression users want to give according to the TPO. In the impression space, this method recommended the users' garments which exist in the shortest distance from clothes in the websites matching the TPO. The shortest distance varies depending on the clothes of each subject. There may be cases where the shortest distance of users' clothes to clothes in the websites matching the TPO is larger than the other subjects. In this case, the method could not recommend the clothes which give others the appropriate impression according to the TPO. It needs to specify the threshold to the shortest distance. From this, the method is expected to be improved in its recommendation accuracy.

\section{B. Clothes to be Supported by others}

In result of the second experiment, the method could recommend clothes which give an impression appropriate to the TPO with $30 \%$ in the precision. This method recommended garments. Therefore, it can be said that garments are made using the clothes which leaned to the user's taste. A possibility that the taste to which the user leaned is parallel with a taste of the partner who meets is low. The recommendation probability is proper. It is necessary to make and recommend garments supported from public people to improve this recommendation precision. For garments of a taste which leaned in the impression space to do a plot, this method has a high possibility that the plot is made the location away from garments of a web which agreed with TPO.

It is necessary to approximate the impression of user's garments to the impression of garments in the website matching the TPO. The solution method creates coordinates incorporate garments using garments in the website that matches the TPO in the user's garments of biased preference. Along the preference of the user, the solution method can create garments supported from public people is not compromised. In the impression space, it is replaced one to clothes approximate the distance between the garments in the website matching the TPO.

The solution method can recommend garments of the impression which is similar to the impression of garments in the website appropriated to TPO. The solution method expected to improve its recommendation accuracy.

\section{CONCLUSION}

This paper proposed a method to recommend garments that give others the appropriate impression users want to give according to the TPO. In the experimental results, this method could recommend garments with which users can give others the appropriate impression with $20 \%$ in the precision.

As future challenges, we improve the extraction method of clothes that matching the TPO. The garments in the website is the coordination public people admit.

\section{REFERENCES}

[1] Itiro Takeuchi, People appearance $90 \%$, Shintyosha, 2005/10

[2] Nobuyuki Sakai, "People tends to judge others by their clothes", Review of living science, Kobe Shoin Women's University of Human Sciences life department, College of life modeling Department laboratory Editing, Vol40, 2009
[3] Vantan communications, "New Fashion business foundation Glossary", channeler 2006

[4] ZOZOTOWN, http://zozo.jp

[5] K. Yamaguchi, M. H. Kiapour, L. E. Ortiz, T. L. Berg, "Retrieving Similar Styles to Parse Clothing" IEEE Transactions on Pattern Analysis and Machine Intelligence, Volume: 37, Issue: 5, 2015

[6] S.kim, "Integration of Environmental Contexts and Personal Factors for Coordinating Garments": An Environmental User Interface Paradigm to Enrich User Interactions, 2009, ACM, Proceedings of the 47th Annual Southeast Regional Conference, 49-55

[7] A. Sato, K.Watanabe, M. Yasumura and J. Rekimoto "suGATALOG: Fashion coordi- nation system that supports users to choose everyday fashion with clothed pictures." Human-Computer Interaction. Towards Intelligent and Im plicit Interaction. Springer Berlin Heidelberg, 2013. 112-121

[8] V. Jagadeesh, R. Piramuthu, A. Bhardwaj, W. Di, N. Sundaresan, "Large Scale Visual Recommendations from Street Fashion Images" $K D D, 2014$ http://dx.doi.org/10.1145/2623330.2623332

[9] S.Kobayashi, "Revised version Psychology of attire", I • K Corporation, 2007

[10] Y.Shirota, "Recommendation Functions by Using Consumer's Kansei on the e-Stylist System", GEM bulletin, 17, pp.53-64, (2003). 